\title{
POLARIZATION SPECTROGRAM OF BIVARIATE SIGNALS
}

\author{
Julien Flamant $^{\natural *} \quad$ Pierre Chainais ${ }^{\natural} \quad$ Nicolas Le Bihan ${ }^{\dagger}$ \\ ${ }^{\natural}$ Univ. Lille, CNRS, Centrale Lille, UMR 9189 - CRIStAL, 59000 Lille, France \\ ${ }^{\dagger}$ CNRS/GIPSA-Lab, Grenoble, France
}

\begin{abstract}
Bivariate signals are commonly processed with the usual Fourier Transform, using methods such as the rotary spectrum analysis. We show that bivariate signals can be efficiently processed using the Quaternion Fourier transform. A bivariate counterpart of the analytic signal is introduced, the quaternion embedding of a complex signal. It leads to identify natural parameters describing polarization properties, amplitude and phase of the signal. The properties of the quaternion short-term Fourier transform are studied and the polarization spectrogram is introduced. A synthetic example illustrates the relevance of the proposed approach.
\end{abstract}

Index Terms - bivariate signal, time-frequency analysis, Stokes parameters, quaternion embedding, polarization spectrogram

\section{INTRODUCTION}

Bivariate signals are encountered in many applications, including oceanography, radar, optics or acoustics. They describe motions in the 2D plane, meaning that a bivariate signal can be represented as a time-evolving vector of $\mathbb{R}^{2}$ or equivalently as the complex-valued signal $x(t)=u(t)+\boldsymbol{i} v(t)$.

Many authors have used augmented representations to extract geometric or polarization properties [1-5] for nonstationary bivariate signals. Bivariate extensions of the Empirical Mode Decomposition have been proposed [6,7], and bivariate instantaneous moments have also been introduced recently [8]. Existing methods rely on the use of the classical complex Fourier Transform (FT). However the classical FT of complex signals has no Hermitian symmetry, thus precluding the direct use of standard time-frequency tools such as the analytic signal.

We show that the Quaternion Fourier Transform (QFT), an alternate definition of the FT, makes it possible to extend time-frequency representations in an elegant manner for bivariate signals. We define the quaternion embedding of a

Corresponding author. julien.flamant@phd.ec-lille.fr

Nicolas Le Bihan's research was supported by the ERA, European Union, through the International Outgoing Fellowship (IOF GeoSToSip 326176) program of the 7 th PCRD.

This work was partly supported by the CNRS, GDR ISIS, within the SUNSTAR interdisciplinary research program. complex signal. It permits to identify instantaneous geometrical parameters, amplitude and phase for simple (monocomponent) signals. Then, using a Quaternion Short-Term Fourier Transform (Q-STFT) a time-frequency representation of bivariate signals is introduced to process multicomponent bivariate signals. This Q-STFT yields to a well-defined spectrogram, where ridges carry instantaneous polarization properties. Section 5 illustrates the relevance of the approach on a synthetic example.

The approach proposed in this article to analyze bivariate signals is an alternative to the rotary spectrum analysis [4]. It consistently generalizes known concepts from univariate nonstationary signal processing. In addition, it provides geometrical interpretation in a dedicated time-frequency representation, the polarization spectrogram.

\section{QUATERNION FOURIER TRANSFORM OF BIVARIATE SIGNALS}

\subsection{Quaternion algebra}

The set of quaternions, denoted $\mathbb{H}$ forms a 4-dimensional algebra with basis $\{1, \boldsymbol{i}, \boldsymbol{j}, \boldsymbol{k}\}$, where $\boldsymbol{i}, \boldsymbol{j}, \boldsymbol{k}$ are imaginary units such that $\boldsymbol{i}^{2}=\boldsymbol{j}^{2}=\boldsymbol{k}^{2}=-1$. Fundamental relations are

$$
\boldsymbol{i j}=\boldsymbol{k}, \boldsymbol{i j}=-\boldsymbol{j i}, \boldsymbol{i j k}=-1,
$$

and subsequent cyclic permutations. Multiplication in $\mathbb{H}$ is non-commutative, thus for $p, q \in \mathbb{H}, p q \neq q p$ in general. Any quaternion $q$ can be written as

$$
q=a+b \boldsymbol{i}+c \boldsymbol{j}+d \boldsymbol{k},
$$

with $a, b, c, d \in \mathbb{R}$. The quaternion conjugate of $q$ is $\bar{q}=a-$ $b \boldsymbol{i}-c \boldsymbol{j}-d \boldsymbol{k}$. Its modulus is $|q|^{2}=q \bar{q}=\bar{q} q=a^{2}+b^{2}+c^{2}+d^{2}$. Involutions with respect to (w.r.t.) $\boldsymbol{i}, \boldsymbol{j}, \boldsymbol{k}$ are given by

$$
\bar{q}^{i}=-\boldsymbol{i} q \boldsymbol{i}, \bar{q}^{j}=-\boldsymbol{j} q \boldsymbol{j}, \bar{q}^{\boldsymbol{k}}=-\boldsymbol{k} q \boldsymbol{k} .
$$

We also introduce the combination between involution w.r.t. $j$ and conjugation like

$$
q^{* \boldsymbol{j}} \triangleq \overline{(\bar{q})}^{\boldsymbol{j}}=\overline{\left(\bar{q}^{\boldsymbol{j}}\right)}=q=a+b \boldsymbol{i}-c \boldsymbol{j}+d \boldsymbol{k} .
$$

Quaternions encompass complex numbers. One can construct complex subfields of $\mathbb{H}$ isomorphic to $\mathbb{C}$, e.g. $\mathbb{C}_{\boldsymbol{i}} \equiv \operatorname{span}\{1, \boldsymbol{i}\}$ 
or $\mathbb{C}_{\boldsymbol{j}}=\operatorname{span}\{1, \boldsymbol{j}\}$. Polar forms of quaternions exist. A particularly useful one is the Euler polar form [9]

$$
q=|q| \exp (\boldsymbol{i} \theta) \exp (-\boldsymbol{k} \chi) \exp (\boldsymbol{j} \varphi),
$$

for $q \in \mathbb{H}$ and with $(\theta, \chi, \varphi) \in[-\pi, \pi[\times[-\pi / 4, \pi / 4] \times$ $[-\pi / 2, \pi / 2]$. Explicit decomposition is given in $[9,10]$. More about quaternions can be found in $[11,12]$.

\subsection{Quaternion Fourier Transform}

Let $x \in L^{2}(\mathbb{R}, \mathbb{H})$. Its Quaternion Fourier Transform (QFT) is given by

$$
X(\omega)=\int x(t) \exp (-\boldsymbol{j} \omega t) \mathrm{d} t,
$$

whereas the inverse QFT reads

$$
x(t)=\frac{1}{2 \pi} \int X(\omega) \exp (\boldsymbol{j} \omega t) \mathrm{d} \omega .
$$

The QFT differs from the standard Fourier transform in two ways. The position of the exponential is crucial due to the non-commutative product in $\mathbb{H}$, with a right-sided convention chosen here. The QFT has a different axis (here $j$ instead of $\boldsymbol{i}$ ). The QFT defined in (6) inherits most properties of the classical Fourier transform. Parseval and Plancherel's theorems exist, meaning that the QFT is an isometry of $L^{2}(\mathbb{R}, \mathbb{H})$. Gabor-Heisenberg uncertainty principle can be extended to the QFT case. It highlights the fact that the QFT obeys the same time-frequency localisation limitations as the classical FT.

Bivariate signals can be represented as complex $\left(\mathbb{C}_{i^{-}}\right.$ valued) signals. For such signals, the QFT has two important properties. First, it gives a quaternion-valued spectral representation, $X(\omega) \in \mathbb{H}$, to any complex signal. Second, the QFT exhibits a particular symmetry

$$
X(-\omega)=-i X(\omega) \boldsymbol{i}=\overline{X(\omega)}^{i},
$$

called the $i$-Hermitian symmetry. These key properties are detailed in [10].

\section{QUATERNION EMBEDDING}

\subsection{Definition}

The $i$-hermitian symmetry (8) of the QFT of complex signals shows that negative frequencies carry no information about the signal. It permits the construction of the quaternion embedding of a complex signal, by simply suppressing negative frequencies of the spectrum. The quaternion embedding of a complex signal is

$$
x_{+}(t)=2 \int_{0}^{+\infty} X(\omega) \exp (\boldsymbol{j} \omega t) \mathrm{d} \omega,
$$

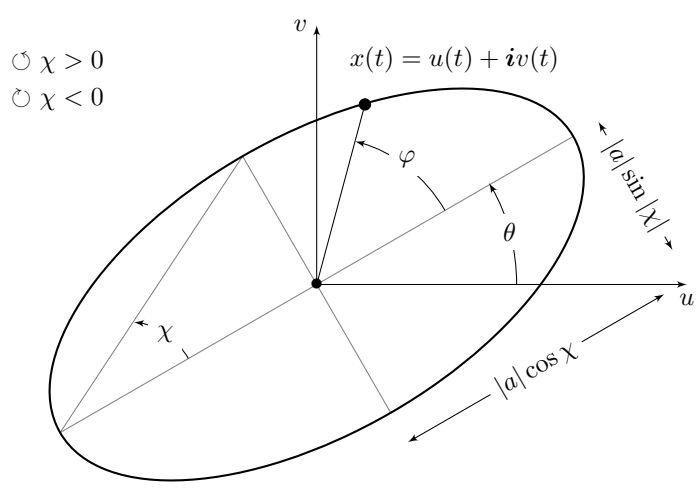

Fig. 1. Geometrical parameters of the bivariate signal $x(t)$ identified using the Euler polar form of its quaternion embedding.

where the multiplicative factor 2 guarantees that $x_{+}(t)$ and $x(t)$ have the same energy. The quaternion embedding $x_{+}(t)$ is a $\mathbb{H}$-valued signal. It is a direct bivariate counterpart of the usual analytic signal, constructed following the same lines.

\subsection{Instantaneous amplitude, ellipticity and phase}

A similar construction of $x_{+}(t)$ was described in [13], with different definitions of instantaneous attributes. Here we propose to interpret $x_{+}(t)$ using its Euler polar form (5) which reads:

$$
x_{+}(t)=a(t) \exp [-\boldsymbol{k} \chi(t)] \exp [\boldsymbol{j} \varphi(t)]
$$

with $a(t) \in \mathbb{C}_{\boldsymbol{i}}, \chi(t) \in[-\pi / 4, \pi / 4]$ and $\varphi(t) \in[-\pi / 2, \pi / 2]$. This decomposition is unique. The original signal $x(t)$ is simply recovered by projecting $x_{+}(t)$ onto $\mathbb{C}_{\boldsymbol{i}}$ as

$$
x(t)=a(t)[\cos \varphi(t) \cos \chi(t)+i \sin \varphi(t) \sin \chi(t)] .
$$

Eq. (11) is a direct bivariate analogue to the univariate AMFM model $x(t)=a(t) \cos [\varphi(t)]$. The quaternion embedding associates a canonical triplet $[a(t), \chi(t), \varphi(t)]$ to any complex signal. This triplet can be interpreted under some usual restrictions [14] adapted to the bivariate case. In brief, we assume that $\varphi(t)$ varies much faster than $a(t)$ and $\chi(t)$ and that $x(t)$ is locally monochromatic.

The quantity $\varphi(t)$ is called the instantaneous phase of $x(t)$, and its derivative $\varphi^{\prime}(t)$ is the instantaneous frequency. Terms $a(t)$ and $\chi(t)$ are respectively called the instantaneous complex amplitude and the instantaneous ellipticity of $x(t)$.

Fig. 1 depicts the ellipse traced out in time by the model (11) when $a(t)=a=|a| \exp (i \theta)$ and $\chi(t)=\chi$ are constant. The value of $|a|$ scales the ellipse. The ellipse orientation is given by $\theta$, whereas its shape is controlled by $\chi$. For $\chi=0$, the ellipse becomes a line segment, whereas extreme cases $\chi= \pm \pi / 4$ describe a circle. The phase $\varphi(t)$ gives the instantaneous position of $x(t)$ in the ellipse, while the sign of $\chi$ gives the direction of rotation. When $a(t)$ and 
$\chi(t)$ are slowly varying the ellipse depicted in Fig. 1 becomes an instantaneous ellipse. The ellipse parameters then meaningfully describe the instantaneous polarization properties of the non-stationary bivariate signal $x(t)$.

The quaternion embedding suffers from the same limitations as the analytic signal. The canonical triplet does not provide useful information when the signal is multicomponent. This limitation is now addressed using the Quaternion Short-Term Fourier Transform (Q-STFT).

\section{QUATERNION SHORT TERM FOURIER TRANSFORM}

Let $g$ be a real and symmetric normalized window, with $\|g\|=1$. For $u, \xi \in \mathbb{R}$, its time-frequency shifted version is

$$
g_{u, \xi}(t)=g(t-u) \exp (\boldsymbol{j} \xi t)
$$

The functions $g_{u, \xi}(t)$ define time-frequency-polarization atoms. The definition of $g_{u, \xi}$ is classical: the term polarization solely indicates that the atoms are $\mathbb{C}_{j}$-valued, rather than $\mathbb{C}_{i}$-valued. The resulting Q-STFT of $x$ is given by

$$
S x(u, \xi)=\int x(t) g(t-u) e^{-j \xi t} \mathrm{~d} t .
$$

The following fundamental theorem proves that the QSTFT is invertible and that energy related quantities are preserved. The proof of this theorem can be found in [10].

Theorem 1 (Inversion formula and energy conservation). Let $x \in L^{2}(\mathbb{R}, \mathbb{H})$. Then the inversion formula reads

$$
x(t)=\frac{1}{2 \pi} \iint S x(u, \xi) g_{u, \xi}(t) \mathrm{d} \xi \mathrm{d} u,
$$

and the energy of $x$ is conserved,

$$
\int|x(t)|^{2} \mathrm{~d} t=\frac{1}{2 \pi} \iint|S x(u, \xi)|^{2} \mathrm{~d} u \mathrm{~d} \xi
$$

as well as the polarization properties of $x$ :

$$
\int x(t) x(t)^{* j} \mathrm{~d} t=\frac{1}{2 \pi} \iint S x(u, \xi) S x(u, \xi)^{* j} \mathrm{~d} u \mathrm{~d} \xi
$$

This theorem demonstrates two things. It extends classical results (see [15]), i.e. Eq. (14) and Eq. (15), to the Q-STFT setting. Moreover Eq. (16) shows that another quantity is preserved by the Q-STFT, related to the polarization content or geometric content of the signal. The quantity $S x(u, \xi) S x(u, \xi)^{* j}$ is called the polarization spectrogram of $x$. It is $\mathbb{H}$-valued and is closely related to Stokes parameters, widely used in optics to describe the polarization of electromagnetic waves [16]. Namely one has

$$
S x(u, \xi) S x(u, \xi)^{* \boldsymbol{j}}=S_{1}(u, \xi)+\boldsymbol{i} S_{2}(u, \xi)-\boldsymbol{k} S_{3}(u, \xi),
$$

where $S_{1}, S_{2}, S_{3}$ are three Stokes parameters. The last Stokes parameter $S_{0}$ is obtained by $S_{0}=\sqrt{S_{1}^{2}+S_{2}^{2}+S_{3}^{2}}$, which is the usual spectrogram of $x$. These parameters are related to the instantaneous complex amplitude and ellipticy the following way

$$
\begin{aligned}
& S_{0}=|a|^{2}, \quad S_{1}=|a|^{2} \cos 2 \chi \cos 2 \theta, \\
& S_{2}=|a|^{2} \cos 2 \chi \sin 2 \theta, \quad S_{3}=|a|^{2} \sin 2 \chi,
\end{aligned}
$$

where the $u, \xi$ dependence has been omitted for sake of simplicity.

Two equivalent time-frequency-polarization representations are then possible. One can represent the usual timefrequency energy density $|S x(u, \xi)|^{2}$, and obtain instantaneous orientation and ellipticity from the ridges of the Q-STFT. Ridges are given by the local maxima of the timefrequency energy density. The second representation is given by the three time-frequency Stokes parameters $S_{1}, S_{2}, S_{3}$ obtained from eq. (17). Both representations imply the simultaneous inspection of three figures. These two representations are equivalent. They are called the polarization spectrogram of $x(t)$.

The theoretical analysis of energy localization in the timefrequency plane, the so called ridge analysis, can be carried out using classical techniques. Using an asymptotic approach [17] it is shown in [10] that the Q-STFT concentrates on the instantaneous frequency line $\xi_{\text {ridge }}(u)=\varphi^{\prime}(u)$. Under mild conditions, one has on the ridge

$$
S x\left(u, \xi_{\text {ridge }}(u)\right)=x_{+}(u) \operatorname{Corr}_{g}^{x}(u)
$$

where the corrective factor $\operatorname{Corr}_{g}^{x}(u)$ takes values in $\mathbb{C}_{j}$. Eq. (19) shows that on the ridge the Q-STFT is simply given by the quaternion embedding of the signal, up to a $\mathbb{C}_{j}$-valued corrective term on the right. It depends on the window $g$ and on the derivatives of the instantaneous frequency $\varphi^{\prime}(t)$. Hence the polarization properties (instantaneous orientation and ellipticity) of the signal are directly readable on the ridge.

\section{EXAMPLES}

Now, we illustrate the performances of the Q-STFT. A synthetic bivariate signal $x(t)$ is made of a superposition of three components: a linear chirp with zero ellipticity and evolving orientation; a quadratic chirp of constant orientation and changing ellipticity; a time-localized signal with constant ellipticity and orientation. The signal is defined for $t \in[0,1]$ by $N=1024$ equispaced samples. The Q-STFT is computed using a Hanning window of size 101 samples, providing a good time-frequency resolution.

Fig. 2 depicts the two equivalent polarization spectrograms of $x(t)$. Fig. 2a, 2b and 2c depict the three timefrequency Stokes parameters. The three Stokes parameters provide an interpretable view of time-frequency-polarization properties of the three components of the signal. The Stokes 


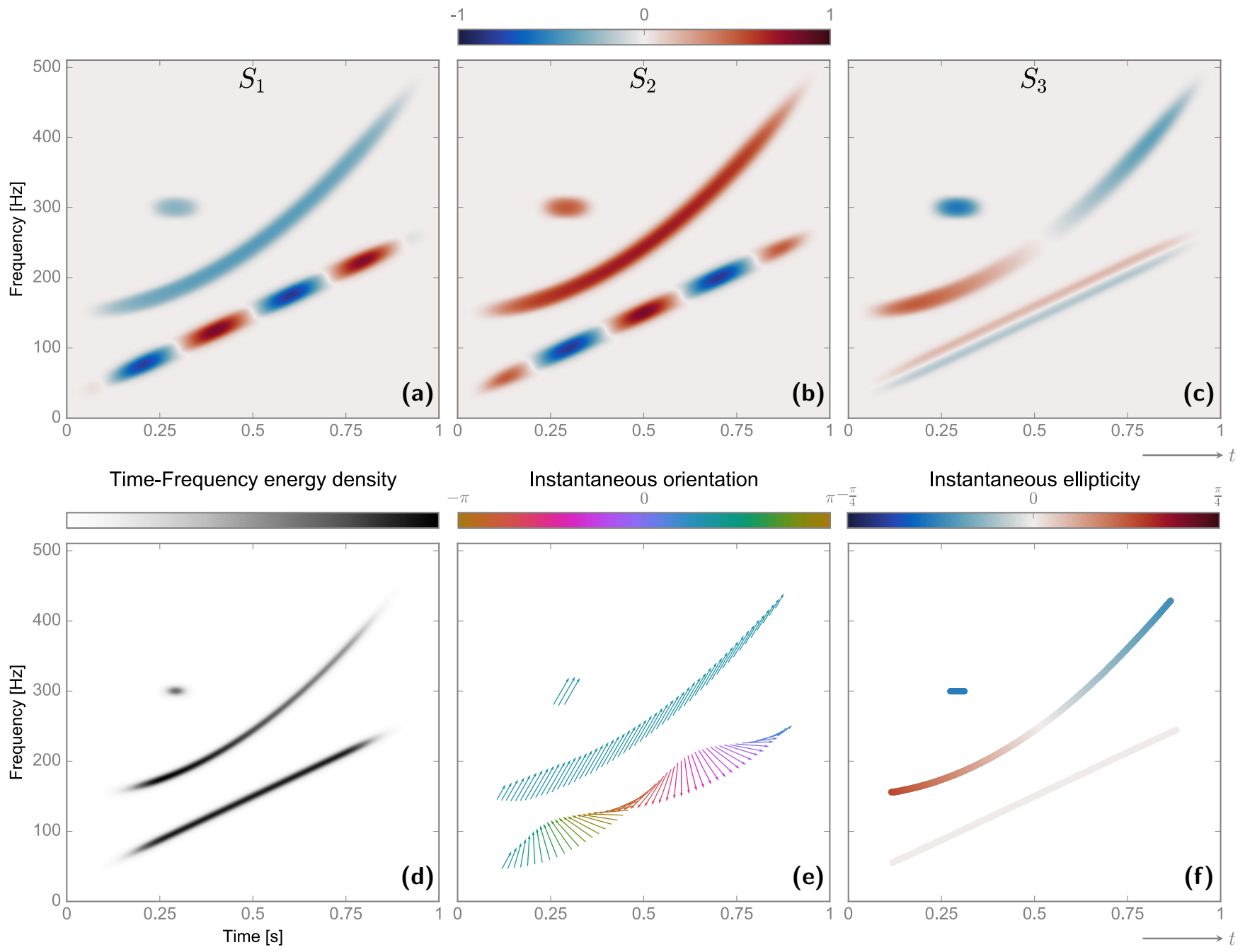

Fig. 2. Synthetic signal and its two equivalent polarization spectrograms. (a), (b) and (c) Stokes parameters in the timefrequency plane. (d) Time-frequency energy density (e) instantaneous orientation (f) instantaneous ellipticity on the ridges.

parameters $S_{1}, S_{2}$ and $S_{3}$ have been normalized by $S_{0}$, so that they provide meaningful geometrical information. While $S_{3}$ is directly an image of the ellipticity, the orientation has to be recovered by simultaneously inspecting the three Stokes parameters, as shown by expressions in (18). One can see in fig. 2c the instantaneous ellipticity of each component, e.g. the quadratic chirp is evolving from left-circular polarization to right-circular polarization. The other two component show constant ellipticity. The periodic alternation of colors due to sign changes in $S_{1}, S_{2}$ in fig. 2a and $2 \mathrm{~b}$ show that the linear chirp component exhibits a rotating orientation.

Fig. $2 d, 2 e$ and $2 f$ show the time-frequency energy density, instantaneous orientation and ellipticity, respectively. The time-frequency energy density in fig. $2 \mathrm{~d}$ also permits the identification of the three components. By extracting the ridge, one gets in fig. $2 \mathrm{e}, 2 \mathrm{f}$ the instantaneous orientation and ellipticity of each component.

\section{CONCLUSION}

The proposed approach extends classical results to the case of bivariate signals using the QFT, and is very general. For monocomponent signals, we introduce the quaternion embedding of a complex signal, which allows to identify instantantaneous geometrical, amplitude and phase quantities. Multicomponent signals are addressed using the Q-STFT. An important theorem shows that the Q-STFT provides an invertible representation and that both energetical and geometrical quantities are preserved. This leads to the definition of a time-frequency representation with a strong physical meaning, called the polarization spectrogram. Numerical implementations are straightforward, of same complexity as the standard FFT algorithm. The presented results have potential applications in identification and separation of components in bivariate signal processing. 


\section{REFERENCES}

[1] Patrick Rubin-Delanchy and Andrew T. Walden, "Kinematics of complex-valued time series," IEEE Transactions on Signal Processing, vol. 56, no. 9, pp. 41894198, 2008.

[2] Peter J. Schreier, "Polarization ellipse analysis of nonstationary random signals," IEEE Transactions on Signal Processing, vol. 56, no. 9, pp. 4330-4339, 2008.

[3] A Serroukh and A T Walden, "Wavelet scale analysis of bivariate time series II: Statistical properties for linear processes," Journal of Nonparametric Statistics, vol. 13, no. 1, pp. 37-56, 2000.

[4] A T Walden, "Rotary components, random ellipses and polarization: a statistical perspective," Philosophical Transactions of the Royal Society of London A: Mathematical, Physical and Engineering Sciences, vol. 371, no. 1984, pp. 20110554, 2013.

[5] Peter J. Schreier and Louis L. Scharf, "Stochastic TimeFrequency Analysis Using the Analytic Signal: Why the Complementary Distribution Matters," IEEE Transactions on Signal Processing, vol. 51, no. 12, pp. 30713079, 2003.

[6] Toshihisa Tanaka and Danilo P Mandic, "Complex Empirical Mode Decomposition," IEEE Signal Processing Letters, vol. 14, no. 2, pp. 101-104, 2007.

[7] G Rilling, P Flandrin, P Goncalves, and J M Lilly, "Bivariate empirical mode decomposition," Ieee Signal Processing Letters, vol. 14, no. 12, pp. 936-939, 2007.

[8] Jonathan M Lilly and Sofia C Olhede, "Bivariate instantaneous frequency and bandwidth," IEEE Transactions on Signal Processing, vol. 58, no. 2, pp. 591-603, 2010.

[9] Thomas Bulow and Gerald Sommer, "Hypercomplex signals-a novel extension of the analytic signal to the multidimensional case," IEEE Transactions on Signal Processing, vol. 49, no. 11, pp. 2844-2852, 2001.

[10] Julien Flamant, Nicolas Le Bihan, and Pierre Chainais, "Time-frequency analysis of bivariate signals," http://arxiv.org/abs/1609.02463, 2016.

[11] John Horton Conway and Derek A Smith, On quaternions and octonions: their geometry, arithmetic, and symmetry, 2003.

[12] Todd A Ell, Nicolas Le Bihan, and Stephen J Sangwine, Quaternion Fourier transforms for signal and image processing, John Wiley \& Sons, 2014.
[13] Nicolas Le Bihan, Stephen J Sangwine, and Todd A Ell, "Instantaneous frequency and amplitude of orthocomplex modulated signals based on quaternion fourier transform," Signal Processing, vol. 94, pp. 308-318, 2014.

[14] Bernard Picinbono, "On instantaneous amplitude and phase of signals," IEEE Transactions on Signal Processing, vol. 45, no. 3, pp. 552-560, 1997.

[15] Stephane Mallat, A wavelet tour of signal processing: the sparse way, Academic press, 2008.

[16] Max Born and Emil Wolf, Principles of optics: electromagnetic theory of propagation, interference and diffraction of light, CUP Archive, 2000.

[17] Nathalie Delprat, Bernard Escudié, Philippe Guillemain, Richard Kronland-Martinet, Ph Tchamitchian, and Bruno Torresani, "Asymptotic wavelet and gabor analysis: extraction of instantaneous frequencies," IEEE transactions on Information Theory, 1992. 